\title{
Comparison of bone remodelling around short stem and conventional straight stem in total hip replacement: a prospective randomized radiographic and dual-energy X-ray absorptiometric study
}

\author{
Lacko Marek $^{1}$, Filip V ${ }^{1}$, Gharaibeh A ${ }^{1}$, Lackova A ${ }^{2}$, Folvarsky $\mathrm{M}^{1}$, Zamborsky $\mathrm{R}^{3}$ \\ Department of Orthopedics and Traumatology of Locomotors Apparatus, Medical Faculty of P.J. Safarik \\ University and University Hospital of L. Pasteur, Kosice, Slovakia. marek.lacko@upjs.sk
}

\begin{abstract}
OBJECTIVE: The aim of study was to evaluate periprosthetic bone mineral density (BMD) changes of proximal femur, osseointegration and clinical outcomes after implantation of short-stemmed and conventional straight-stemmed prostheses.

METHODS: This prospective, randomized study included 50 patients with unilateral total hip replacement.

The patients were randomized into 2 cohorts: patients with a cementless short stem Metha $(n=25)$

and patients with a cementless conventional straight stem Bicontact evaluated as the control $(n=25)$.

Periprosthetic BMD changes were measured using a DEXA performed at one-week, 3-monts, 6-months and 1-year follow up. Clinical evaluation with Harris hip score (HHS) and radiographic assessment were performed through a 1 year follow up.

RESULTS: Compared to 1-week postoperative assessment, there were differences in BMD changes between the groups at the final follow-up in all ROls, with statistical significances in ROI 1, 2, 3, 6 and 7. The loss of periprosthetic BMD in all ROIs around straight stems at each time-point was observed. There was a tendency towards a regain of BMD in all ROls at 1-year follow-up compared to the 3-months postoperative assessment with the short stems. Less pronounced bone loss was observed around the short stems that the straight stems in ROI $1(-2.9 \% \%$ vs $-16.2 \%), 5(-4.7 \%$ vs $-8.9 \%)$ and $7(-8.6 \%$ vs $-20 \%)$. The periprosthetic BMD exceeded baseline values in the short stem cohort in ROI $2(+4.4 \%$ vs $-5 \%), 3(+5.6 \%$ vs $-2.5 \%)$ and 6 $(+4.3 \%$ vs $-10 \%)$. All stems had a radiographically stable fixation. Stress shielding-related bone resorption was markedly lower in the short stem cohort. The HHS score was comparable between the two cohorts. CONCLUSION: The implant-specific stress shielding altered the proximal loading condition for both stems; however, the results of this study suggest a more physiological strain distribution with the short stems versus the straight stems (Tab. 3, Fig. 3, Ref. 25). Text in PDF www.elis.sk

KEY WORDS: total hip replacement, DEXA, Short stem, straight stem, periprosthetic BMD.
\end{abstract}

\section{Introduction}

Total hip replacement (THR) is one of the most effective procedure in orthopaedic surgery. THR in younger patients has become more common over last decades. The risk for a revision

${ }^{1}$ Department of Orthopedics and Traumatology of Locomotors Apparatus, Medical Faculty of P.J. Safarik University and University Hospital of L. Pasteur, Kosice, Slovakia, ${ }^{2}$ Department of neurology, University hospital of L. Pasteur, Kosice, Slovakia, and ${ }^{3}$ Department of Orthopedics, Medical Faculty of Comenius University, Bratislava, Slovakia

Address for correspondence: M. Lacko, Ass Prof, MD, PhD, Department of Orthopedics and Traumatology of Locomotors Apparatus, Medical Faculty of P.J. Safarik University and University Hospital of L. Pasteur, Trieda SNP 1, SK-040 01 Kosice, Slovakia.

Phone: +421.908619734 , Fax: +421.5.53200

Acknowledgements: The work was supported by the Scientific Grant Agency of the Ministry of Education, Science, Research and Sports of the Slovak Republic - VEGA no.1/0598/20. surgery is higher due to longer life expectancy and activity level of these patients (1).

Short femoral stems have been introduced in primary hip arthroplasty with the aim to preserve trochanteric and femoral neck bone stock and to restore more physiological loading in the proximal femur reducing the risk of stress shielding (2). Therefore, these shorter femoral implants may be alternative to cementless conventional straight stems in younger and more active patients.

The stress shielding is determined with changes in the distribution of loads on host bone after THR and is mainly a consequence of different elasticity of the implant and the periprosthetic bone (3). Stress shielding usually involves the proximal femur by reducing the mechanical strength and ability to withstand the transmitted loads, potentially leading to aseptic loosening and periprosthetic fatigue fractures (4).

The area of implant fixation and load transfer can be determined by assessing periprosthetic bone remodelling, with periprosthetic bone mineral density (BMD) changes measured with 
dual-energy X-ray absorptiometry (DEXA) $(5,6,7)$. The most of the dynamic remodelling processes are determinable within first year after THR, followed by minimal changes in periprosthetic bone quality $(8,9,10)$.

The purpose of this study was to evaluate implant-specific BMD changes of proximal femur, osseointegration and clinical outcomes after the implantation of the short-stemmed femoral prostheses Metha and the conventional straight-stemmed prostheses Bicontact. Our hypothesis was that the short-stemmed implants provide more physiological bone remodelling with a lower periprosthetic bone loss, with a good fixation and clinical outcomes.

\section{Material and methods}

This single-center, prospective, randomized, comparative study included a consecutive series of 50 patients ( 29 males and 21 females; the mean age 54.9 years; range 45 to 65 years), who underwent an unilateral total hip replacement at the University Hospital of Louis Pasteur in Kosice for the primary or secondary osteoarthritis between December 2014 and January 2019.

The inclusion criteria compromised the patients with the endstage unilateral osteoarthritis of hip (Kellgren-Lawrence grade 3 and 4), age ranging from 40 to 65 years old, body mass index between 18 and $35 \mathrm{~kg} / \mathrm{m}^{2}$, with a bone stock suitable for cementless hip arthroplasty - femur type Dorr A or B (11). Exclusion criteria were hip dysplasia, previous surgery in the same hip, femoral fracture, osteoporosis (DEXA T-score $\leq 2.5$ ) and other metabolic bone diseases, drugs affecting bone metabolism and contralateral THR within the study period (12 months).

The patients were randomized into 2 cohorts for comparison: (1) patients with a cementless trochanter-sparing short stem Metha $(\mathrm{n}=25)$ and (2) patients with a cementless conventional standard straight stem Bicontact evaluated as the control $(n=25)$. Online statistical computing web programming was used (www.graphpad. com/quickcalcs) to randomize the subjects into 2 groups with an equal sample size. The CONSORT chart illustrating the enrolment of patients is shown in Figure 1.

The study protocol was approved by the Faculty of medicine of P.J. Safarik University in Kosice Ethics Committee (approval number: 8/2013). A written informed consent was obtained from each patient. The study was conducted in accordance with the principles of the Declaration of Helsinki.

\section{Surgical procedure}

All THRs were performed at a single institute by four experienced orthopaedic surgeons, through a standard anterolateral approach. In all cases, the Plasmacup (BBraun, Aesculap, Tuttlingen, Germany) press-fit acetabular component with a ceramic insert was used. Patients received either a cementless short stem Metha or a cementless straight stem Bicontact. The ceramic (Biolox Delta) head was used. In this study, only the stems were examined and not the cups.

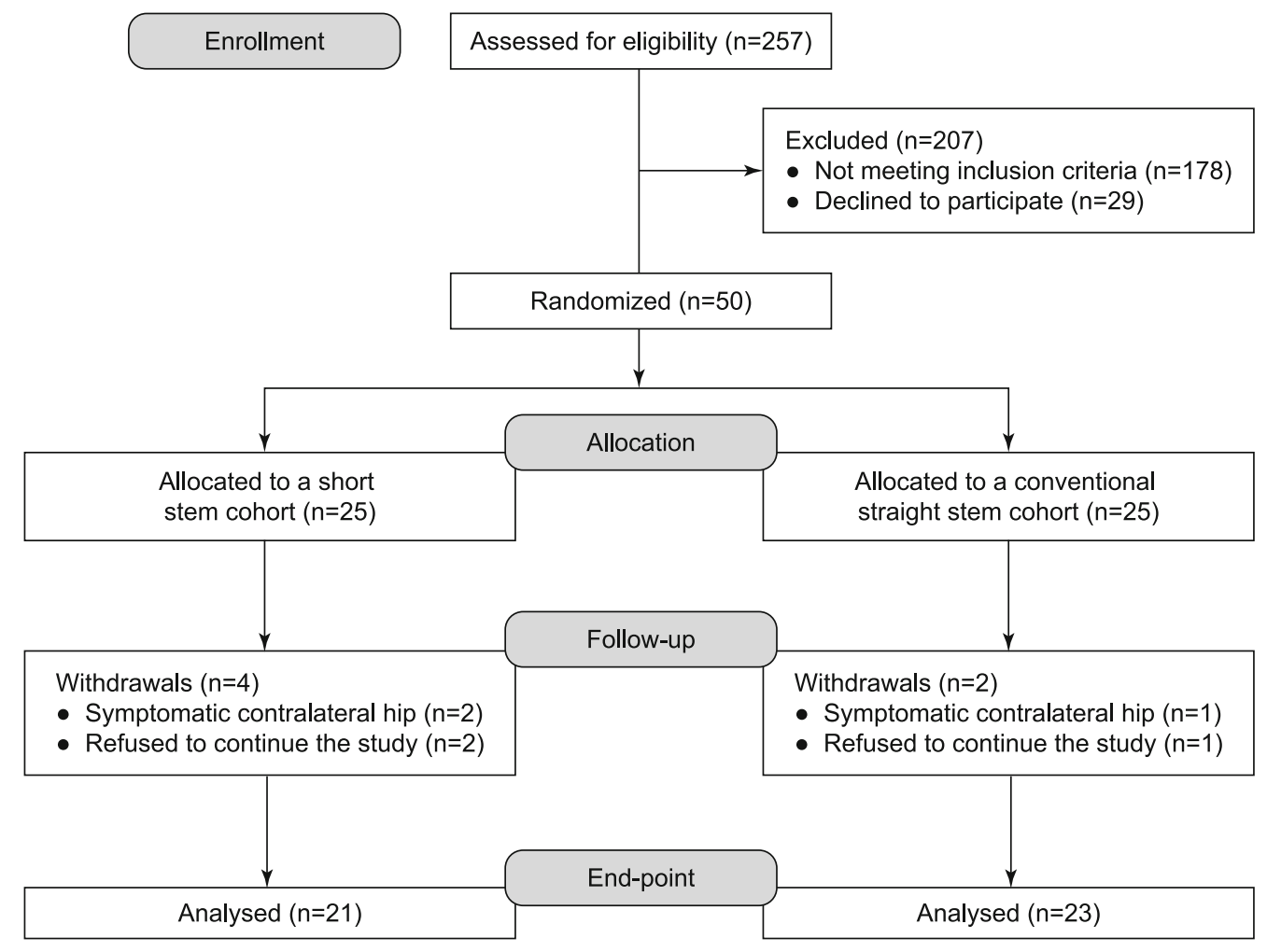

Fig. 1. Consolidated standards of reporting trials (CONSORT) flow diagram used to describe the grouping and flow of patients in the trial. 


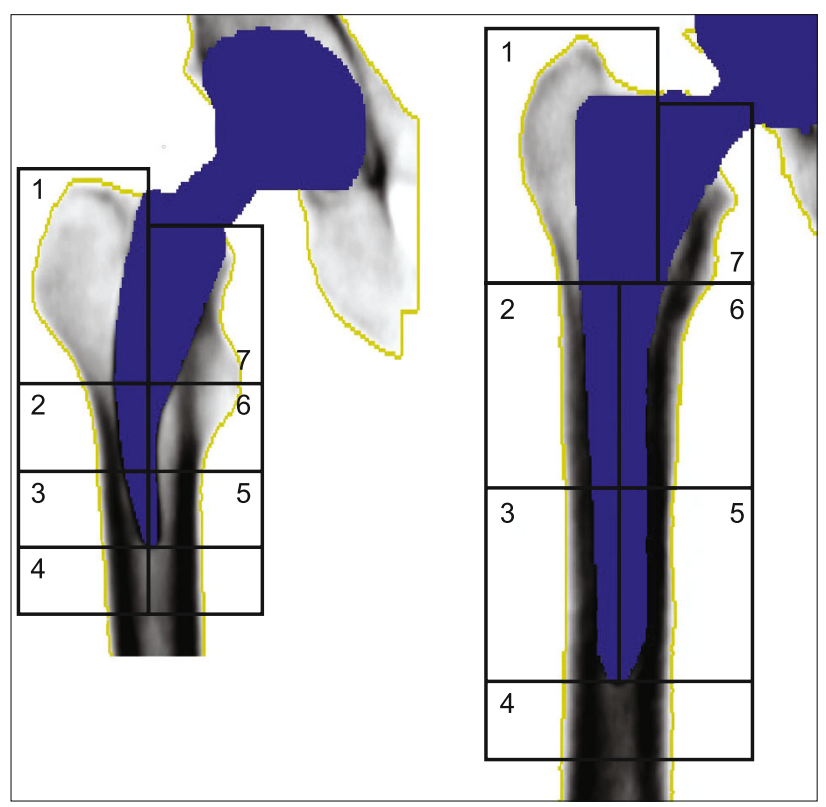

Fig. 2. The Metha stem and Bicontact stem with standardized regions of interest (ROI) in a screenshot from DEXA scanner.

Metha short stem (BBraun, Aesculap, Tuttlingen, Germany) is made of a titanium forged alloy $\left(\mathrm{Ti}_{6} \mathrm{Al}_{4} \mathrm{~V}\right)$. The stem has a proximal rough titanium, plasma-sprayed, microporous coating. An additional $20 \mu \mathrm{m}$ dicalcium phosphate dihydrate $\left(\mathrm{CaHPO}_{4} \times 2 \mathrm{H}_{2} \mathrm{O}\right)$ layer is applied electrochemically. The stem is anchored metaphyseally within the closed ring of the femoral neck.

Bicontact straight stem (BBraun, Aesculap, Tuttlingen, Germany) is made of a titanium forged alloy $\left(\mathrm{Ti}_{6} \mathrm{Al}_{4} \mathrm{~V}\right)$. The stem has a proximal microporous pure titanium Plasmapore coating. A flat, rectangular stem shape, lateral fixation fins and a dorsal antirotational wing give the stem primary stability and proximal force transmission exclusively in the intertrochanteric region. Distal pressfit is expressively not intended with this design.

All the patients were allowed a partial weight bearing using two crutches postoperatively for 2 months.

\section{Demographic data}

The basic characteristics of individual groups were based on the expression of the mean age, male to female ratio, body mass index (BMI), classification of perioperative risk according to the American Society of Anaesthesiologists (ASA), and the distributions of proximal femoral bone type according to Dorr's classification.

\section{DEXA assessment}

DEXA assessment was performed at one-week, 3-months, 6-months and 1-year follow-up. Bone mineral density (BMD) $\left(\mathrm{g} / \mathrm{cm}^{2}\right)$ of operated hip was measured with the Lunar Prodigy Dualenergy X-ray Absorptiometry device (General Electrics Healthcare Medical Systems, Europe, Belgium) at each assessment. All the scans were performed in a supine position with the leg in a jig to secure a constant internal rotation of $20^{\circ}$. Contralateral hip and lumbar spine were scanned preoperatively to exclude osteoporosis (12). The BMD data collected at 1 week after surgery served as the baseline value for comparison and changes were expressed in percent. All the DEXA scans and analyses were performed by the same observer using the metal-removal hip-scan mode (Fig. 2). Due to the different femoral component lengths around the straight stems, we used conventional seven regions of interest (ROI) described by Gruen et al (13), around the short femoral stems we used seven modified ROIs $(8,14)$. The quality controls were performed every day for the DEXA equipment to verify system stability.

\section{Clinical assessment}

Clinical evaluation, included the Harris hip score (HHS), was performed pre-operatively and post-operatively at 1-year followup (15). Also, any intra-operative and postoperative complications were recorded.

\section{Radiographic assessment}

The femoral morphology was determined in preoperative radiographs using Dorr's classification (11). Radiological evaluation included anterior-posterior X-rays of operated hip performed in the day of surgery and at 1-year follow up. All radiographs were reviewed by a single observer for subsidence more than $2 \mathrm{~mm}$, osteolytic areas, radiolucent lines around the stem, cortical hypertrophy, calcar resorption and presence of heterotopic ossification. Proximal femoral bone resorption was graded radiologically, with the grade 1 showing atrophy or rounding off of the calcar; the grade 2, loss of density in the calcar region with a preservation of the medial cortex to the level of the lesser trochanter; the grade 3 , loss of density in the calcar region with a loss of the medial cortex to the level of the lesser trochanter; and the grade 4, loss of density in the entire medial cortical wall distal to the level of the lesser trochanter (16).

\section{Statistical analysis}

The descriptive statistics (mean, standard deviation, and proportions) for the continuous study variables were calculated. The Harris hip score and BMD data were compared between the 2 groups with the Student independent t-test. If the normality test failed, the Mann-Whitney test was used. Statistical significance was defined at a significance level of $\mathrm{p}<0.05$. SigmaPlot version 12.5 (Systat Software, Inc., San Jose, CA, USA) was used for statistical analyses.

\section{Results}

After 12-months of follow-up, six patients were excluded from the study: the contralateral hip became symptomatic in three patients and three patients refused to continue the study. 44 patients completed the final 1-year follow-up. There were no differences in the patient demographic parameters between the groups (Tab. 1).

\section{DEXA outcome}

BMD findings were significantly different between the 2 groups at 6-months and 1-year follow-up. 
Tab. 1. Patient demographics.

\begin{tabular}{lccc}
\hline Parameter & $\begin{array}{c}\text { Short-stem } \\
(\mathrm{n}=21)\end{array}$ & $\begin{array}{c}\text { Conventional stem } \\
(\mathrm{n}=23)\end{array}$ & $\mathrm{p}$ \\
\hline Age $($ years $)$ & $52.9(9.4)$ & $55.8(5.3)$ & $\mathrm{NS}$ \\
BMI $\left(\mathrm{kg} / \mathrm{m}^{2}\right)$ & $27.1(3.8)$ & $28.5(3.4)$ & $\mathrm{NS}$ \\
Female/male & $9 / 12$ & $10 / 13$ & $\mathrm{NS}$ \\
ASA $1 / 2 / 3$ (n) & $5 / 15 / 1$ & $4 / 17 / 2$ & $\mathrm{NS}$ \\
Dorr class A/B/C $(\mathrm{n})$ & $15 / 6 / 0$ & $14 / 9 / 0$ & $\mathrm{NS}$ \\
\hline
\end{tabular}

Values are expressed as the mean, with a standard deviation in parentheses; $\mathrm{p}$ determined with Student's t-test; NS = not significant

Tab. 2. The mean percentage change in bone mineral density (BMD).

\begin{tabular}{|c|c|c|c|}
\hline Change in BMD & $\begin{array}{l}\text { Short-stem } \\
(n=21)\end{array}$ & $\begin{array}{l}\text { Standard straight stem } \\
(\mathrm{n}=23)\end{array}$ & $\mathrm{p}$ \\
\hline \multicolumn{4}{|l|}{ ROI 1} \\
\hline 3 months & $-7.9(4.8)$ & $-7.3(14.3)$ & NS \\
\hline 6 months & $-6.4(7.6)$ & $-9.9(17.3)$ & NS \\
\hline 12 months & $-2.9(12.7)$ & $-16.2(20.5)$ & 0.031 \\
\hline \multicolumn{4}{|l|}{ ROI 2} \\
\hline 3 months & $-7.5(7.3)$ & $-7.2(6.6)$ & NS \\
\hline 6 months & $-0.5(13.5)$ & $-4.8(10.4)$ & NS \\
\hline 12 months & $4.4(12.9)$ & $-4.5(10.3)$ & 0.049 \\
\hline \multicolumn{4}{|l|}{ ROI 3} \\
\hline 3 months & $-4.1(6.6)$ & $-5.0(4.7)$ & NS \\
\hline 6 months & $-1.0(6.8)$ & $-3.3(5.2)$ & NS \\
\hline 12 months & $5.6(8.9)$ & $-2.5(6.2)$ & 0.003 \\
\hline \multicolumn{4}{|l|}{ ROI 4} \\
\hline 3 months & $-5.8(4.6)$ & $-3.9(7.5)$ & NS \\
\hline 6 months & $-6.5(5.9)$ & $-4.9(5.4)$ & NS \\
\hline 12 months & $-5.3(6.1)$ & $-5.3(9.6)$ & NS \\
\hline \multicolumn{4}{|l|}{ ROI 5} \\
\hline 3 months & $-7.2(5.8)$ & $-6.9(9.4)$ & NS \\
\hline 6 months & $-6.7(8.0)$ & $-7.1(10.3)$ & NS \\
\hline 12 months & $-4.7(9.8)$ & $-8.9(13.2)$ & NS \\
\hline \multicolumn{4}{|l|}{ ROI 6} \\
\hline 3 months & $-3.8(6.3)$ & $-6.6(6.9)$ & NS \\
\hline 6 months & $1.7(7.4)$ & $-6.0(9.1)$ & NS \\
\hline 12 months & $4.3(10.8)$ & $-10.1(9.6)$ & $<0.001$ \\
\hline \multicolumn{4}{|l|}{ ROI 7} \\
\hline 3 months & $-11.1(6.0)$ & $-13.1(12.2)$ & NS \\
\hline 6 months & $-10.5(8.2)$ & $-15.0(3.3)$ & 0.012 \\
\hline 12 months & $-8.6(11.9)$ & $-20.2(14.5)$ & 0.018 \\
\hline
\end{tabular}

$\mathrm{ROI}=$ range of interest; Values are expressed as the mean, with a standard deviation in parentheses; $p$ determined with Student's t-test; NS = not significant

After 3 months, a decrease in BMD was detected in all the 7 ROIs for both implants versus the 1-week postoperative assessment. There was no significant difference in BMD changes between the groups at this point of time.

A reduction of BMD was observed at 6 months, compared to 1-week results in all the ROIs for both cohorts, except for ROI 6 in short stem cohort. Continuing reduction of BMD was found in the short stem cohort in ROI 4. In the straight stem cohort, continuous reduction of BMD was observed in ROI 1, 4, 5 and 7. There were significant differences in BMD changes among the groups in the ROI 7.

Compared with 1-week postoperative assessment, there was a decrease of BMD in all the ROIs at 1-year follow-up in the conventional stem cohort. The decrease in periprosthetic BMD was
Tab. 3. Preoperative and postoperative Harris hip score (HHS).

\begin{tabular}{lccc}
\hline Parameter & $\begin{array}{c}\text { Short-stem } \\
(\mathrm{n}=21)\end{array}$ & $\begin{array}{c}\text { Conventional stem } \\
(\mathrm{n}=23)\end{array}$ & $\mathrm{p}$ \\
\hline HHS preoperative & $37.4(12.5)$ & $35.1(10.3)$ & NS \\
HHS 1-year follow up & $88.3(9.5)$ & $86.8(8.6)$ & NS \\
\hline
\end{tabular}

Values are expressed as the mean, with a standard deviation in parentheses; $\mathrm{p}$ determined with Student's t-test; NS = not significant

most pronounced in ROI 1 and 7. In the short stem cohort, reduction of BMD has been found in ROI 1, 4, 5 and 7. The reduction in BMD was lower for short stems compared to the standard stems. It was remarkable that BMD exceeded baseline values in ROI 2, 3 and 6 in the short stem cohort. There was a significant difference in BMD changes between the groups at 1-year follow-up in all the ROI, except for the ROI 4 and 5. The DEXA outcomes are presented in Table 2 and Figure 3.

\section{Radiological outcome}

Radiological examination showed no subsidence greater than $2 \mathrm{~mm}$ or radiolucent lines, so all the stems were declared stable.

No hip with a short stem had a pedestal formation, distal cortical hypertrophy, or cortical thinning. Twelve (52\%) of 23 hips with a standard femoral stem had a pedestal formation, and 18 (78 \%) had developed a distal cortical hypertrophy. Stress shielding-related bone resorption was markedly lower in the short stem cohort than in the standard stem cohort. Two (10\%) of 21 hips with a short stem had the grade 1 bone loss. Fifteen hips (63\%) with the straight stem had the grade 1 (4 hips), 2 ( 8 hips) or the grade 3 bone loss ( 3 hips).

No patient had the grade 3 or 4 heterotopic ossification in either group.

\section{Clinical outcome}

There were significant increases in HHS score in both the groups for all the patients at 1-year follow-up compared to preoperative values. There were no significant differences between the two groups in HHS score either before surgery or at 1-year follow up (Tab. 3).

No intra-operative or postoperative complications were observed.

\section{Discussion}

Short-stemmed femoral implants were designed to achieve more proximal and physiological load transfer in the femoral metaphysis in order to avoid stress shielding and to preserve metaphyseal bone. This is associated with a potentially decreased risk of aseptic loosening, periprosthetic fracture and easier revision surgery, which was considered the major advantage compared to the conventional straight stems (2). Studies examining a strain distribution after the implantation of short femoral stems reported conflicting results regarding the achievement of selective proximal load transfer $(17,18)$. 


\section{8-554}
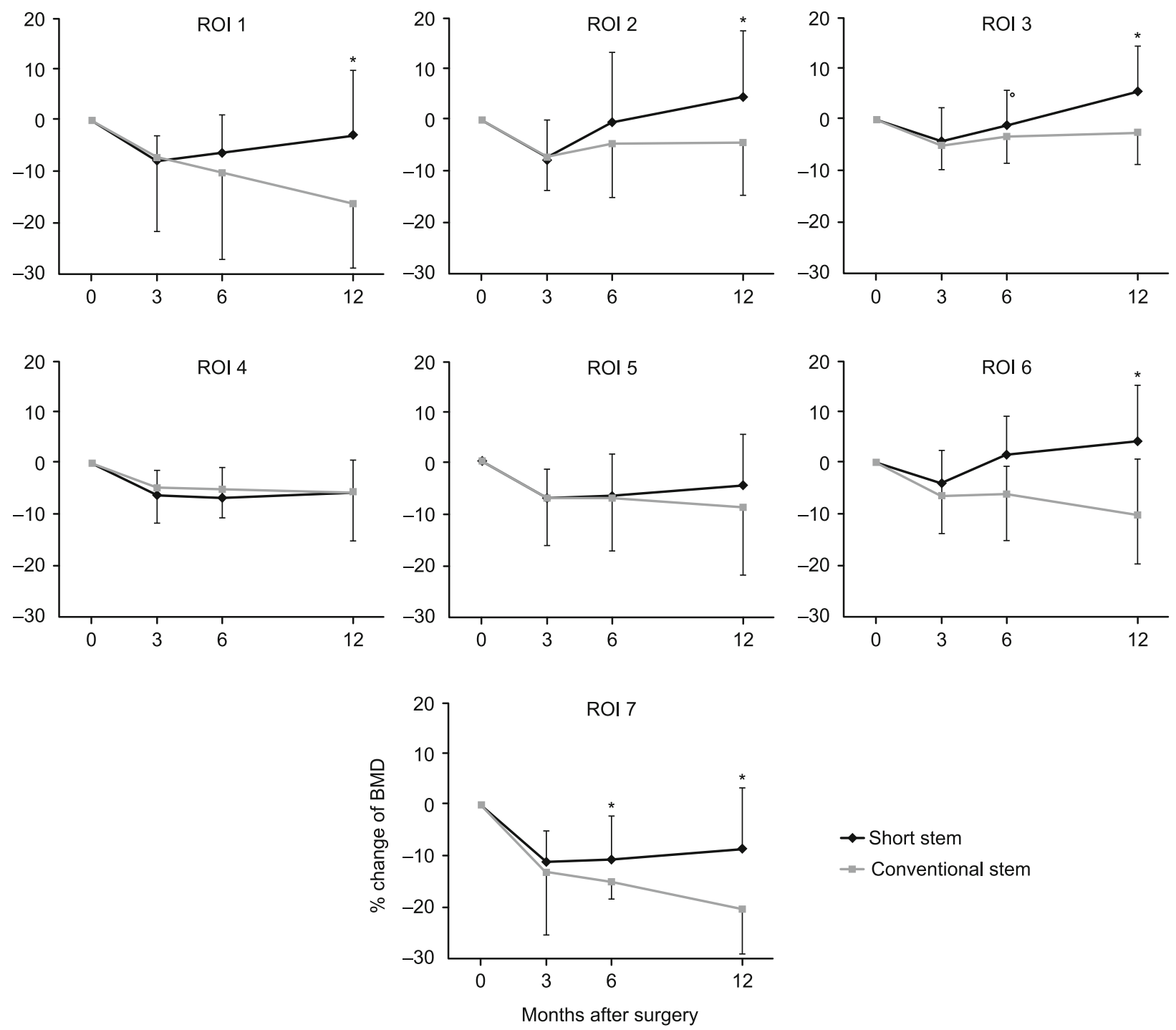

Fig. 3. Mean percentage change in BMD. ROI = range of interest; Error bars indicate standard deviation; Differences were analyzed with Student's t-test; ${ }^{*} \mathbf{p}<\mathbf{0 . 0 5}$.

Periprosthetic BMD changes following the femoral stem implantation detect the presence of implant-specific stress shielding. The most reliable measure of periprosthetic BMD, with a high degree of accuracy and reproducibility is DEXA with periprosthetic BMD changes expressed as the percentage change in comparison with immediate postoperative values $(5,6,8,19)$.

In this study, periprosthetic BMD changes within 1 postoperative year were compared between the short stem and the standard straight stem cohorts. Compared with 1-week postoperative assessment, there were differences in BMD changes between the groups at the final follow-up in all the ROIs, with the statistical significances in ROI 1, 2, 3, 6 and 7. There was a loss of periprosthetic BMD in all the ROIs around the straight stems at each point of time. On the other hand, there was a tendency towards regaining of BMD in all the ROIs at 1-year follow-up compared to the 3-months postoperative assessment with the short stems. Less pronounced bone loss was observed around the short stems that the straight stems in ROI 1 ( $-2.9 \%$ vs $-16.2 \%), 5(-4.7 \%$ vs $-8.9 \%)$ and $7(-8.6 \%$ vs $-20 \%)$. It was remarkable that BMD exceeded baseline values in the short stem cohort in ROI $2(+4.4 \%$ vs $-5 \%), 3(+5.6 \%$ vs $-2.5 \%)$ and $6(+4.3 \%$ vs $-10 \%)$. However, the implant-specific stress shielding altered the proximal loading condition for both stems, these findings suggest a more physiological load transfer with the short stems versus the straight stems.

Comparing the results of this study with the previously published findings is challenging due to methodical differences such as the varying location and size of ROIs or different types of implants. We found only 2 studies examining the BMD changes of proximal femur after the Metha stem implantation. Lerch et al (8) identified an increase of BMD one year after THR in ROI 2 
$(+1 \%)$ and $6(+8 \%)$ and a significant decrease of BMD in ROI $1(-12 \%)$ and $7(-10 \%)$. Brickmann et al (17) identified a maximal decrease of BMD in upper calcar region (ROI $7 ;-11 \%$ ) and great trochanteric region (ROI $1 ;-9 \%$ ). They found an increased BMD only in lower calcar region (ROI $6 ;+8 \%$ ). Similarly, in our study, increased BMD was found in ROI $2(+4.4 \%)$ and 6 $(+4.3 \%)$, moreover also in ROI $3(+5.6 \%)$, however, a significantly smaller reduction in ROI $1(-2.9 \%)$ and $7(-8.6 \%)$ was observed. These results suggest only a small to moderate bone loss in the great trochanteric and calcar regions after implantation of the Metha stems one year postoperatively. None of these two studies compared BMD changes around short stems with the conventional straight stems as the controls. In other studies, evaluating periprosthetic BMD changes after the conventional straight stems $(20,21)$, proximal BMD loss has been quoted as high as 12 to $30 \%$, with increasing BMD under the tip of femoral stem (ROI 4). These findings are comparable with the results of this study.

We found that $58 \%$ of the conventional stems had a pedestal formation, $78 \%$ had a distal cortical hypertrophy and $65 \%$ had a bone atrophy in the calcar region. No pedestal formation or distal cortical hypertrophy and only a mild bone atrophy occurred in $10 \%$ of short-stemmed THRs. These findings confirmed that a distal load transfer with typical stress shielding phenomena was associated with the conventional stems. On the other hand, an absence of a diaphyseal anchoring of the short femoral stem can challenge the primary stability required for osseointegration of the implant. Several authors pointed out a greater postoperative migration of short-stemmed femoral prostheses $(22,23,24)$. In this study cohorts, all the femoral components had radiographically stable fixation, with no migration.

The clinical results of this study showed, that one year after THR, HHS score was comparable between the two cohorts, with patients achieving a significant improvement. This finding is in agreement with other studies $(8,17)$. Adequate long-term results are not proven for short femoral stems (2).

The present study has several limitations. One limitation is the relative short follow-up. On the other hand, several studies indicated that there is an acute phase of bone remodelling within first postoperative year, followed by only minimal changes in periprosthetic $\operatorname{BMD}(8,9,10,25)$. Another limitation is the different implant lengths used in this study, which make a direct comparison difficult. Furthermore, we also did not analyse the influence of patients-specific parameters (such as age, gender, BMI) on periprosthetic BMD changes.

The strength of this study is its prospective nature with a randomization to equal comparison cohorts with similar demographics.

In conclusion, THR with the short stem Metha and the conventional straight stem Bicontact showed excellent clinical results and reliable osseointegration over a short follow-up period. The implant-specific stress shielding altered the proximal loading condition for both stems; however, the results of this study suggest a more physiological strain distribution in proximal femur with the short stems versus the straight stems. Furthermore, the long-term evaluation is necessary to confirm the clinical value of the preservation of proximal femoral bone stock after the implantation of the short-stemmed femoral prostheses, particularly in terms of aseptic loosening and improved conditions for revision surgery.

\section{References}

1. Bayliss LE, Culliford D, Monk AP et al. The effect of patient age at intervention on risk of implant revision after total replacement of the hip or knee: a population-based cohort study. Lancet 2017; 389 (10077): 1424-1430.

2. Loppini M, Grappiolo G. Uncemented short stems in primary total hip arthroplasty: The state of the art. EFORT Open Rev 2018; 3 (5): 149-159.

3. Sumner DR. Long-term implant fixation and stress-shielding in total hip replacement. J Biomech 2015; 48 (5): 797-800.

4. Apostu D, Lucaciu O, Berce C, Lucaciu D, Cosma D. Current methods of preventing aseptic loosening and improving osseointegration of titanium implants in cementless total hip arthroplasty: a review. J Int Med Res 2018; 46 (6): 2104-2119.

5. Parchi PD, Cervi V, Piolanti $\mathbf{N}$ et al. Densitometric evaluation of periprosthetic bone remodelling. Clin Cases Miner Bone Metab 2014; 11(3): 226-231.

6. Venesmaa PK, Kroger HP, Miettinen HJ, Jurvelin JS, Suomalainen OT, Alhava EM. Monitoring of periprosthetic BMD after uncemented total hip arthroplasty with dual-energy X-ray absorptiometry - a 3-year follow-up study. J Bone Miner Res 2010; 16 (6): 1056-1061.

7. Sessa G, Costarella L, Puma Pagliarello C et al. Bone mineral density as a marker of hip implant longevity: a prospective assessment of a cementless stem with dual-energy X-ray absorptiometry at twenty years. Int Orthop 2019; 43 (1): 71-75.

8. Lerch M, von der Haar-Tran A, Windhagen H, Behrens BA, Wefstaedt P, Stukenborg-Colsman CM. Bone remodelling around the Metha short stem in total hip arthroplasty: a prospective dual-energy X-ray absorptiometry study. Int Orthop 2012; 36 (3): 533-538.

9. Brodner W, Bitzan P, Lomoschitz F et al. Changes in bone mineral density in the proximal femur after cementless total hip arthroplasty. A five-year longitudinal study. J Bone Joint Surg Br 2004; 86 (1): 20-26.

10. Panisello JJ, Herrero L, Herrera A et al. Bone remodelling after total hip arthroplasty using an uncemented anatomic femoral stem: a threeyear prospective study using bone densitometry. J Orthop Surg 2006; 14 (1): $32-37$.

11. Dorr LD. Total hip replacement using the APR system. Tech Orthop 1986; 1 (3): 22-34.

12. Brazdilova K, Dlesk A, Koller T, Killinger Z, Payer J. Vitamin D deficiency - a possible link between osteoporosis and metabolic syndrome. Bratisl Med J 2012; 113 (7): 412-416.

13. Gruen TA, McNeice GM, Amstutz HC. Modes of failure" of cemented stem-type femoral components: a radiographic analysis of loosening. Clin Orthop Rel Res 1979; 141: 17-27.

14. Falez F, Casella F, Panegrossi G et al. Perspectives on metaphyseal conservative stems. J Orthop Traumatol 2008; 9 (1): 49-54.

15. Mahomed NN, Arndt DC, McGrory BJ, Harris WH. The Harris hip score: comparison of patient self-report with surgeon assessment. J Arthroplasty 2001; 16 (5): 575-580. 


\section{8-554}

16. Engh CA, McGovern TF, Bobyn JD et al. A quantitative evaluation of periprosthetic bone-remodelling after cementless total hip arthroplasty. J Bone Joint Surg Am 1992; 74 (7): 1009-1020.

17. Brinkmann V, Radetzki F, Delank KS, Wohlrab D, Zeh A. A prospective randomized radiographic and dual-energy X-ray absorptiometric study of migration and bone remodelling after implantation of two modern short-stemmed femoral prostheses. J Orthop Traumatol 2015; 16 (3): 237-243.

18. Gotze C, Ehrenbrink J, Ehrenbrink H. Is there a bone-preserving bone remodelling in short-stem prosthesis? DEXA analysis with the Nanos total hip arthroplasty. Z Orthop Unfall 2010; 148 (4): 398-405.

19. Miznerova E, Hlavaty T, Koller T, Toth J, Holociova K, Huorka M, Killinger Z, Payer J. The prevalence and risk factors for osteoporosis in patients with inflammatory bowel disease. Bratisl Med J 2013; 114(8): 439-445.

20. Boden HS, Skoldenberg OG, Salemyr MO, Lundberg HJ, Adolphson PY. Continuous bone loss around a tapered uncemented femoral stem: a long-term evaluation with DEXA. Acta Orthop 2006; 77 (6): 877-885.

21. Lerch M, Kurtz A, Windhagen $H$, Bouguecha A, Behrens BA, Wefstaedt P, Stukenborg-Colsman CM. The cementless Bicontact stem in a prospective dual-energy X-ray absorptiometry study. Int Orthop 2012; 36 (11): 2211-2217.
22. Salemyr M, Muren O, Ahl T et al. Lower periprosthetic bone loss and good fixation of an ultra-short stem compared to a conventional stem in uncemented total hip arthroplasty. Acta Orthop 2015; 86 (6): $659-666$.

23. Floerkemeier T, Budde S, Lewinski GV, Windhagen H, HurSchler C, Schwarze M. Greater early migration of a short-stem total hip arthroplasty is not associated with an increased risk of osseointegration failure: 5th-year results from a prospective RSA study with 39 patients, a followup study. Acta Orthop 2020; 91 (3): 266-271.

24. Schaer MO, Finsterwald M, Holweg I et al. Migration analysis of a metaphyseal-anchored short femoral stem in cementless THA and factors affecting the stem subsidence. BMC Musculoskelet Disord 2019; 20 (1): 604.

25. Freitag T, Hein MA, Wernerus D, Reichel H, Bieger R. Bone remodelling after femoral short stem implantation in total hip arthroplasty: 1-year results from a randomized DEXA study. Arch Orthop Trauma Surg 2016; 136 (1): 125-130. 